\title{
Port performance evaluation tool based on microsimulation model
}

\author{
Jzolanda Tsavalista Burhani ${ }^{1, *}$, Febri Zukhruf ${ }^{1}$, and Russ Bona Frazila ${ }^{1}$ \\ ${ }^{1}$ Institut Teknologi Bandung, Civil Engineering Department, 40132 Bandung, Indonesia
}

\begin{abstract}
As port performance is becoming correlative to national competitiveness, the issue of port performance evaluation has significantly raised. Port performances can simply be indicated by port service levels to the ship (e.g., throughput, waiting for berthing etc.), as well as the utilization level of equipment and facilities within a certain period. The performances evaluation then can be used as a tool to develop related policies for improving the port's performance to be more effective and efficient. However, the evaluation is frequently conducted based on deterministic approach, which hardly captures the nature variations of port parameters. Therefore, this paper presents a stochastic microsimulation model for investigating the impacts of port parameter variations to the port performances. The variations are derived from actual data in order to provide more realistic results. The model is further developed using MATLAB and Simulink based on the queuing theory.
\end{abstract}

\section{Introduction}

Maritime transportation plays a major role in national and international trade, and it has a significant impact to national economic growth [1]. For instance, more than $90 \%$ of international cargo move through seaports, and $80 \%$ of seaborne cargo ships use containerized vessels, which has increased by $3.4 \%$ and $5.3 \%$ annually [2]. In response to this growth, the container terminal (CT) operators thus strive to stay competitive by offering competitive services and land transportation services [3]. In addition, in order to meet the container demands effectively and efficiently, the port performance evaluation tool becomes overly essential. It functions to answer several issues relating to the port performances: to know the effectivity and efficiency of its operation; to compare the current performance and the past performance; to adjust its targets for future periods; etc. [4].

CT operations have been considered as complex systems which involve the interactions of many resources, entities, and activities, namely, the ships arriving, the multiple quay cranes (QC) and the rubber tyred gantry cranes (RTGC) loading/unloading, the trucks delivering, and the security gates open/closing [5]. Due to the complexity and scale of operations, it is relatively challenging to develop the mathematical model for analyzing the whole CT system, and hence, the existing research mostly considers the single aspect of CT [6]. The simulation modelling (SM) is then prospectively used as a tool for demonstrating, evaluating, and optimizing the system performance [7], which is practically developed using the computer program as it is implemented in this paper.
SMs have been widely used and applied in the modelling, planning, management, and analysis of CTs $[6,8,9,10,11,12]$. Started in 1960s, SM remains popular as a decision-making tool for various purposes, especially for complex systems that cannot be represented by the analytical models [13]. Dragović et al. [14] and Angeloudis et al. [15] provided the complete reviews about the SMs in ports and CTs. The investigation of $\mathrm{CT}$ performance has been treated using various simulation models, which are coded in the different languages: SIMPLE++ [16], ARENA [5, 17, 18, 19], Matlab [20], AweSim [21], AutoMod. [22], PORTSIM [23]. Mainly, previous researches present the simulation models for analyzing and evaluating the terminal performance at current configuration. In addition, preceding papers also show that the simulation models can be built from a sequence of operational procedures for determining the port performances in the various environment and heterogeneous cases within the different points of view. Relating to the CT operations, Carteni et al. [24] proposed a discrete-event simulation model and applied it to the Salerno CT to investigated the effects of different hypotheses on handling equipment models.

Practically, SMs can be divided into some categories: microscopic, macroscopic, stochastic, and deterministic models. The microscopic (operational) model takes into account the detail behaviour of vehicles and the traffic control infrastructure in order to replicate the dynamic systems of terminal. In contrast, the macroscopic (strategic) model, which has a lower fidelity, is developed based on the aggregate information [15]. Furthermore, the stochastic model considers the stochastic features by generating random numbers to

Corresponding author: tjzolanda@ymail.com 
capture the uncertainty of real-world systems. The rest of SM, which does not contain any probabilistic components, are called deterministic models [8, 13].

This paper then develops a microsimulation model for evaluating the CT performance. For capturing the stochastic phenomena of $\mathrm{CT}$, this paper also conducts several experiments with different initial seed value using MATLAB and Simulink software. The rest of the paper is organized as follows. In the following section, the system and modelling framework is described. In the third section, the experimental study is conducted for investigating the applicability of model. Finally, in the fourth section, the methodologies, results, and analyses in the paper are summarized.

\section{The simulation model development}

\subsection{System description}

The simulation model developed in this paper considers the whole process of import container movements in the $\mathrm{CT}$. The process starts from the ship unloading process and ends in the administration process in the terminal gate. The container is firstly discharged from a ship, and it is sequentially headed to the berth using QCs. Each ship is practically served by 2 or 3 QCs, where the time required by the QC for serving the container is called as the QC service time. There are various types of QC that possibly influence its service time, however this paper only involves a Panamax QC (P-QC), which is generally used in Indonesia.

The container is then transferred to the truck trailer unit (TTU), which is utilized for transporting it to the container yard (CY) through the horizontal transport area. The TTU service time, which is defined as the time required for transferring container to $\mathrm{CY}$, is evaluated as its performance indicator. Arriving at $\mathrm{CY}$, the container is further stacked by the first RTGC (F-RTGC) until the consignees fulfil the requirements for taking it. The time spent by the container within the CY area is named as the storage service time. If the consignees can pass the requirement, the last RTGC (L-RTGC) is employed for transporting and heading the container to the external truck (XT). The time required by the L-RTGC to serve a container is called L-RTGC service time. This process indicates the completion process at the terminal.

In the next step, the container is transported by XT for leading out of the terminal area through a gate. The time required by the XT to transports the container is called as XT service time. In the final stage, the XT faces the administration process in the gate, which is indicated by the gate service time, before it is distributed to their hinterland area. The total time spent by the container within the $\mathrm{CT}$ area, namely from the P-QC until the gate, is defined as the dwelling time.

The rich picture-based procedures are invoked for constructing the CT system in the simulation model. The procedures contain three main components, namely the structure, process, and each process relationship [25]. Structural aspects describe the body structure of model, which can be illustrated as follows:
1. Entity, is an object that is served in the system: container.

2. Generator, is a unit that produces entities at any given time period: ship.

3. Servers, are units that serve entites in a certain period of time: P-QC, TTU, F-RTGC, L-RTGC, storage area, XT, and gate.

4. Queues, are places for entities to wait before they are served: P-QC queue and storage queue. Queue has its own capacity for serving entities.

5. Attributes of entity, are properties which are owned by the entity: inter-arrival rate (which represent the period of time between the arrival of the entity), waiting time, P-QC service time, TTU service time, F-RTGC service time, storage service time, L-RTGC service time, XT service time, gate service time, and dwelling time.

6. Attributes of system, are properties which are owned by the system: capacity of P-QC queue, capacity of storage queue, length of P-QC queue, and length of storage queue.

The second aspect of procedure is related to the process which occurs in the CT system, such as:

1. The arrival process of the ship carries containers.

2. The waiting process of container for the P-QC service.

3. The transporting process of container from the ship to the TTU using P-QC.

4. The transporting process of container by the TTU along the horizontal transportation area and the transferring process to the F-RTGC at CY.

5. The stacking process of container by F-RTGC in CY.

6. The waiting process of container before being taken by the consignee.

7. The servicing process of container by L-RTGC.

8. The transferring process of storage container to the XT.

9. The transporting process of container to the gate area by the XT.

10. The gate services for XT.

11. The time spent by the container within the CT area since served by P-QC until out of gate.

The relationship among processes is then explained as follows:

1. P-QC service time is affected by the process of transporting container from the ship to the TTU.

2. TTU service time is affected by the transporting and transferring process of container from the TTU to the F-RTGC in CY.

3. F-RTGC service time is affected by the stacking process of container from the TTU to the F-RTGC.

4. Storage service time is affected by the waiting and servicing process of container to be served by LRTGC.

5. L-RTGC service time is affected by the transferring process of container to the XT.

6. XT service time is affected by the transporting process of container from the CY to the gate.

7. Gate service time is affected by the XT service process. 
8. The length of P-QC queue is influenced by the P-QC service time.

9. The length of storage queue is influenced by storage service time.

10. Dwelling time is an acculumation of all existing attributes.

11. Dwelling time is affected by the whole process for handling the import containers.

\subsection{Input and output variables}

There are two types of input variables that affect the system: controllable and uncontrollable input. Controllable input variables are generally described as decision variables in the system, for instance the number of P-QC, the number of TTU, the number of F-RTGC, the number of $\mathrm{XT}$, and the number of gate. Uncontrollable input variables are related to the input values that cannot be controlled, for example, the interarrival rate of a container, the capacity of P-QC queue, the P-QC service time, the TTU service time, the FRTGC service time, the number of storage, the storage service time, the capacity of storage queue, the number of L-RTGC, the L-RTGC service time, the XT service time, and the gate service time.

The outputs are then illustrated by the performances of CT operation, for instance, the number of containers served, the average number of containers which are waiting in each queue, the average time of containers for waiting in each queue, the average service time of each server, the dwelling time of system, and the utilities of equipment.

\subsection{Assumptions of model}

Although the model is developed for representing the actual condition of CT, there are several assumptions used, which can be described as follows:

1. There is no warming up time on the model, and hence a continuous time is assumed.

2. In the beginning of the simulation, it is assumed that there are no containers in the system, and thus the queue is empty and the server is not used (idle).

3. The port is assumed to be operated 24 hours in a day.

4. The replacement of the vessel process is not considered.

5. The queue priority follows the principle of First In First Out (FIFO).

6. The server is always available during the simulation process.

7. The overbrengen process in the container handling is not considered.

8. Empty container handling processes are not considered.

9. The import customs process is not considered.

\subsection{Model transformation}

The model further will be transformed into a programming language i.e. MATLAB and Simulink using
SimEvents blocks [26]. Here are brief explanations of the existing subsystems created in the SM.

1. Container generator subsystem, is a subsystem that regulates containers which is produced in a specific time. Some important parameters in this subsystem, namely, the distribution parameter and the initial seed parameter.

2. Set service time subsystem, is a subsystem that organizes the attributes of the service time. Some important parameters in this subsystem i.e. distribution parameter that controls the distribution of random numbers that we want.

3. Output switch subsystem, is a subsystem that organizes the entites output port selection criteria for the entities departure. Some important parameters in this subsystem, such as, the switching criterion parameter.

4. Enabled gate subsystem, is a subsystem that organizes the entrance arrangement for the entities.

5. Server queue subsystem, is a subsystem that organizes the entities waiting to be served in specified or unspecified time.

6. Single server subsystem, is a subsystem that organizes the service for entities in a certain period of time.

7. Read timer after server subsystem, is a subsystem that organizes the statistic data information on the timer which associated with the arrival of entities.

8. Path combiner subsystem, is a subsystem that organizes the incorporation of entities pathway.

9. Containers sink subsystem, is a subsystem that organizes the acception or rejection of entites.

\section{Experimental study}

In order to test the model's applicability, the experimental study is conducted by considering the actual data from Koja Container Terminal (KCT) relating to the facilities performance (i.e., berth facilities, $\mathrm{CY}$, container handling equipment). The $\mathrm{KCT}$ can handle containers up to 7560 TEUs for import and 7696 TEUs for export. Operating 5 blocking parallel layouts of CY, the KCT also uses the combination of RTGC and TTU for handling such operations. In addition, KCT is also equipped with 7 QCs, 48 head trucks, 60 chassis, 25 RTGCs, 3 reach stackers, and a fire fighting car.

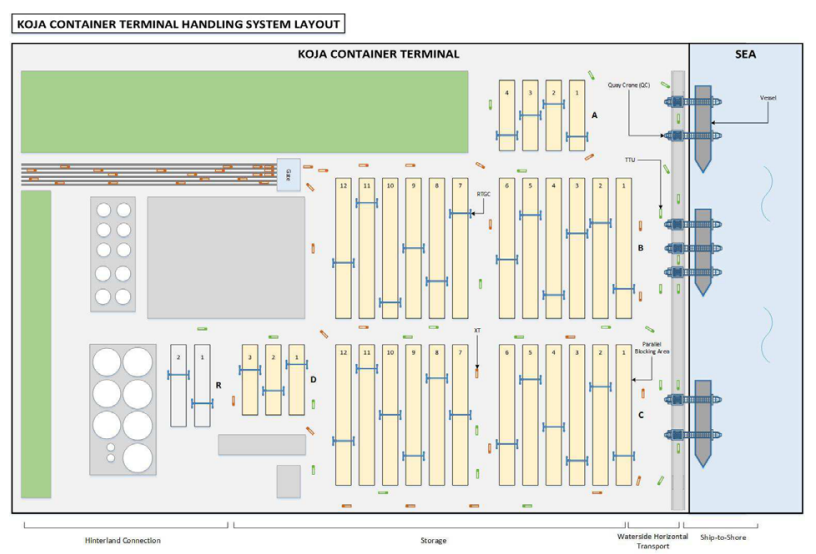

Fig. 1. Koja container terminal layout 
The actual data, which is derived from the KCT operational reports, is then utilized as the simulation inputs: the inter-arrival rate of entities, the capacity of server queue, the number of equipment, and the equipment service time. The characteristics of simulation inputs are summarized in Table 1 , in which the random number is generated by following the triangular distribution.

Table 1. The simulation input

\begin{tabular}{|l|c|c|c|c|c|c|}
\hline \multicolumn{1}{|c|}{ Parameters } & Units & Min & Max & Mode & Mean & Stdev \\
\hline Inter-Arrival Rate of Entities & TEUs/min & 1.00 & 2.00 & 1.00 & 1.00 & 0.00 \\
\hline Capacity of P-QC Queue & TEUs & - & inf & - & - & - \\
\hline P-QC Service Time & $\mathrm{min}$ & 2.00 & 3.00 & 2.50 & 0.00 & 0.00 \\
\hline TTU Service Time & $\mathrm{min}$ & 24.00 & 43.00 & 29.00 & 31.00 & 5.00 \\
\hline F-RTGC Service Time & $\mathrm{min}$ & 5.00 & 8.00 & 7.00 & 6.00 & - \\
\hline Capacity of Storage Queue & TEUs & - & 630.00 & - & - & - \\
\hline Storage Service Time & $\mathrm{min}$ & 70.56 & 180.00 & 93.60 & 11.00 & - \\
\hline L-RTGC Service Time & $\mathrm{min}$ & 5.00 & 8.00 & 7.00 & 6.00 & - \\
\hline XT Service Time & $\mathrm{min}$ & 94.00 & 191.00 & 106.00 & 130.00 & 24.00 \\
\hline Gate Service Time & $\mathrm{min}$ & 1.00 & 3.00 & 2.00 & 2.00 & - \\
\hline
\end{tabular}

Since the container practically needs to spend around 12 days for passing the terminal gate from the ship unloading, this paper then simulates 12 days of unloading operation in KCT. The model implementation is conducted with 20 attempts of different initial seed value for capturing the natural stochastic phenomena of CT. In other words, the experiment tries to imitate 240 days of stochastic terminal operations (see Figure 2).

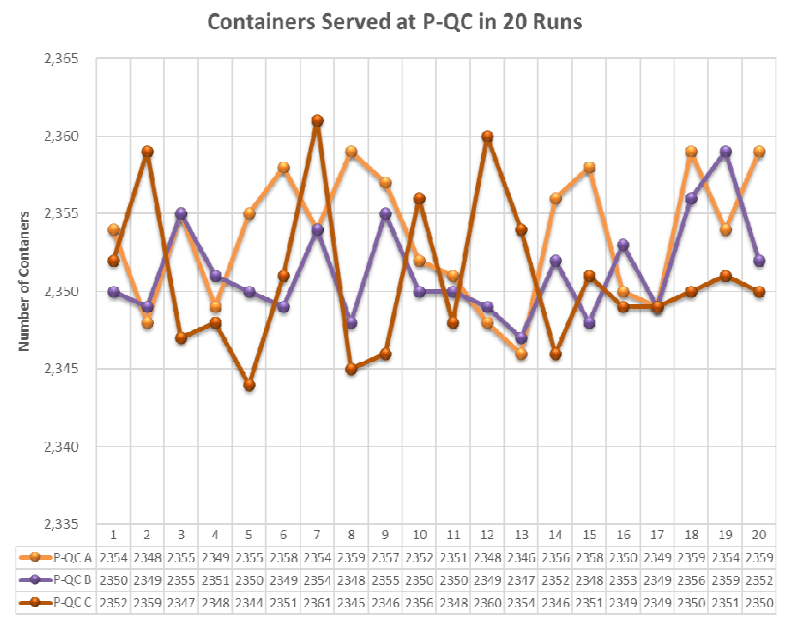

Fig. 2. Number of containers served by P-QC

As the important indicator of port performance, the container queue is firstly elaborated. The experimental results show that the longest queue occurs before the PQC server, which cause the queue for 698 containers (see Table 2). A similar result also occurs in the storage area, which involve 409 containers in queue. The long queue is probably caused by the lack number of equipment compared to the container arrival rate. In contrast, the results show that there is no queue before FRTGC. It indicates that the number of F-RTGC and FRTGC service time is on adequate condition.
Table 2. Number of containers in queue

\begin{tabular}{|l|c|}
\hline \multicolumn{1}{|c|}{ Parameters } & Results (containers) \\
\hline Total number of queue at P-QC & 2094 \\
\hline Average number of queue at P-QC & 698 \\
\hline Total number of queue at storage & 3674 \\
\hline Average number of queue at storage & 409 \\
\hline Total number of queue at F-RT GC & 0 \\
\hline Average number of queue at F-RT GC & 0 \\
\hline
\end{tabular}

Furthermore, the utilization ratio of equipment is also explored. The interesting result is laid on the P-QC, TTU, and storage, where they have a utilization ratio of near one (i.e. $0.999,0.988$ and 0.997 , respectively). This result describes that those servers already face their highest performance for servicing high workloads. On the contrary, the F-RTGC, L-RTGC, XT, and gate results in a low utilization ratio (i.e., 0.691, 0.058, 0.563, 0.039 , respectively). This condition may indicate the server's ability of service is too fast compared to the inter-arrival rate of containers. In addition, it can also be caused by the long deposition on the previous server, which causes in an idle and unproductive server.

\section{Conclusion}

This paper presents a microsimulation model for analyzing the operational performances of CT. The model is developed by utilizing the rich picture-based procedures for imitating the whole of import CT system. For investigating the applicability of model, the actual data of CT is considered in the model. The simulation is conducted by generating several different numbers of initial seed in order to describe a real world system. The experimental results show that the model can identify the several operational problems of $\mathrm{CT}$, which is potentially used as a tool for evaluating the CT performance as well as for managing the CT operation.

\section{References}

1. De Monie, G. Measuring And Evaluating Port Performance And Productivity. UNCTAD monographs on port management, No. 6 (United Nations, 1987). doi:GE.87-56444/0295G

2. Regina et al. Review of Maritime Transport 2015. (United Nations, 2015).

3. Alderton, P. \& Saieva, G. Port Management and Operations. (Informa, 2008).

4. Esmer, S. Performance Measurements of Container Terminal Operations. 10, 238-255 (2008).

5. Kotachi, M., Rabadi, G. \& Obeid, M. F. Procedia Comput. Sci. 20, 229-234 (2013).

6. Huang, S. Y., Hsu, W. J., Chen, C., Ye, R. \& Nautiyal, S. Int. J. Comput. Appl. Technol. 32, 246253 (2008).

7. Law, A. M. \& Kelton, W. D. Simulation Modeling and Analysis 3rd Edition. (McGraw-Hill, 2000).

8. Dragovic, B. \& Zrnic, N. D. A Queuing Model 
Study of Port Performance Evolution. Analele Univ. 'Eftimie Murgu' Resita Fasc. Ing. 2, 65-76 (2011).

9. Gudelj, A., Krčum, M. \& Twrdy, E. Models and Methods for Operations in Port Container Terminals. PROMET - Traffic \& Transportation 22, 43-51 (2010).

10. Legato, P., Mazza, R. M. \& Trunfio, R. SimulationBased Optimization for Discharge/Loading Operations at a Maritime Container Terminal. OR Spectr. 32, 543-567 (2010).

11. Peng-fei, Z., Zi-jian, G. \& Xiang-qun, S. International Conference on Management Science and Engineering 177-182 (IEEE, 2006). doi:10.1109/ICMSE.2006.313837

12. Ramani, K. V. An Interactive Simulation Model for the Logistics Planning of Container Operations in Seaports. Simulation 66, 291-300 (1996).

13. Kelle, P. \& Jin, M. A Simulation Model for Intermodal Freight Transportation in Louisiana. (2015).

14. Dragović, B., Tzannatos, E. \& Park, N. K. Simulation Modelling in Ports and Container Terminals: Literature Overview and Analysis by Research Field, Application Area, and Tool. Flex. Serv. Manuf. J. 1-31 (2016). doi:10.1007/s10696016-9239-5

15. Angeloudis, P. \& Bell, M. G. H. A Review of Container Terminal Simulation Models. Marit. Policy Manag. 38, 523-540 (2011).

16. Yun, W. Y. \& Choi, Y. S. Int. J. Prod. Econ. 59, 221-230 (1999).
17. Kulak, O., Polat, O. \& Guenther, H.-O. 3rd German-Korean Workshop on Container Terminal Management (2008).

18. Kyu Park, N., Dragović, B., Zrnić, N. \& S-H Moon, D. Simulation Approach of Container Terminal Modelling.

19. Park, N.-K. \& Dragović, B. A Study of Container Terminal Planning. FME Trans. 37, 203-209 (2009).

20. Ansorena, I. L., Orive, A. C., Cancelas, N. G. \& Orive, A. C. Marit. Transp. V. Technol. Innov. Res. 448-460 (2012).

21. Nam, K.-C., Kwak, K.-S. \& Yu, M.-S. J. Waterw. Port, Coastal, Ocean Eng. 128, 126-132 (2002).

22. Lau, Y. K. H. \& Lee, M. Y. N. Simulation Study of Port Container Terminal Quay Side Traffic. AsiaSim 5, 227-236 (2007).

23. Nevins, M. R., Macal, C. M., Love, R. J. \& Bragen, M. J. Simulation, Animation and Visualization of Seaport Operations. Simulation 71, 96-106 (1998).

24. Cartenì, A. \& Luca, S. de. Tactical and Strategic Planning for A Container Terminal: Modelling Issues Within A Discrete Event Simulation Approach. Simul. Model. Pract. Theory 21, 123-145 (2012).

25. Daellenbach, H. G. System and Decision Making: $A$ Management Science Approach. (John Wiley \& Sons, Ltd., 1996). doi:10.1002/bdm.3960080407

26. MATLAB \& Simulink: SimEvents 3 User's Guide. (The Mathworks,

Corresponding author: tjzolanda@ymail.com 